\title{
Mycophenolate versus Azathioprine as Maintenance Therapy for Lupus Nephritis
}

\author{
Mary Anne Dooley, M.D., M.P.H., David Jayne, M.D., Ellen M. Ginzler, M.D., \\ M.P.H., David Isenberg, M.D., Nancy J. Olsen, M.D., David Wofsy, M.D., \\ Frank Eitner, M.D., Gerald B. Appel, M.D., Gabriel Contreras, M.D., M.P.H., \\ Laura Lisk, B.Sc., and Neil Solomons, M.D., for the ALMS Group*
}

A BSTRACT

From the Department of Medicine, University of North Carolina, Chapel Hill (M.A.D.); the Renal Unit, Addenbrooke's Hospital, Cambridge (D.J.), and Vifor Pharma, Bagshot Park, Bagshot, Surrey (L.L.) - both in the United Kingdom; the Division of Rheumatology, State University of New York Downstate Medical Center, Brooklyn (E.M.G.); the Department of Medicine, University College, London (D.I.); the Division of Rheumatology, Penn State Milton S. Hershey Medical Center, Hershey, PA (N.J.O.); the Division of Rheumatology and Rosalind Russell Medical Research Center, University of California, San Francisco (D.W.); the Division of Nephrology and Immunology, Rheinisch-Westfälische Technische Hochschule, Aachen University, Aachen, Germany (F.E.); the Department of Nephrology, Columbia University, New York (G.B.A.); the Division of Nephrology and Hypertension, University of Miami Miller School of Medicine, Miami (G.C.); and the Clinical Department, Vifor Pharma (formerly Aspreva Pharmaceuticals), Victoria, Canada (N.S.). Address reprint requests to Dr. Solomons at the Clinical Department, Vifor Pharma, \#1203, 4464 Markham St., Victoria, BC V8Z 7X8, Canada, or at neil.solomons@viforpharma.com.

*Members of the Aspreva Lupus Management Study (ALMS) Group are listed in the Supplementary Appendix, available at NEJM.org.

N Engl J Med 2011;365:1886-95. Copyright $(\odot 2011$ Massachusetts Medical Society.

\section{BACKGROUND}

Maintenance therapy, often with azathioprine or mycophenolate mofetil, is required to consolidate remission and prevent relapse after the initial control of lupus nephritis.

\section{METHODS}

We carried out a 36-month, randomized, double-blind, double-dummy, phase 3 study comparing oral mycophenolate mofetil ( $2 \mathrm{~g}$ per day) and oral azathioprine ( $2 \mathrm{mg}$ per kilogram of body weight per day), plus placebo in each group, in patients who met response criteria during a 6-month induction trial. The study group underwent repeat randomization in a 1:1 ratio. Up to $10 \mathrm{mg}$ of prednisone per day or its equivalent was permitted. The primary efficacy end point was the time to treatment failure, which was defined as death, end-stage renal disease, doubling of the serum creatinine level, renal flare, or rescue therapy for lupus nephritis. Secondary assessments included the time to the individual components of treatment failure and adverse events.

\section{RESULTS}

A total of 227 patients were randomly assigned to maintenance treatment (116 to mycophenolate mofetil and 111 to azathioprine). Mycophenolate mofetil was superior to azathioprine with respect to the primary end point, time to treatment failure (hazard ratio, $0.44 ; 95 \%$ confidence interval, 0.25 to $0.77 ; \mathrm{P}=0.003$ ), and with respect to time to renal flare and time to rescue therapy (hazard ratio, $<1.00 ; \mathrm{P}<0.05$ ). Observed rates of treatment failure were $16.4 \%$ ( 19 of 116 patients) in the mycophenolate mofetil group and $32.4 \%$ (36 of 111) in the azathioprine group. Adverse events, most commonly minor infections and gastrointestinal disorders, occurred in more than $95 \%$ of the patients in both groups $(\mathrm{P}=0.68)$. Serious adverse events occurred in $33.3 \%$ of patients in the azathioprine group and in $23.5 \%$ of those in the mycophenolate mofetil group $(\mathrm{P}=0.11)$, and the rate of withdrawal due to adverse events was higher with azathioprine than with mycophenolate mofetil (39.6\% vs. $25.2 \%, \mathrm{P}=0.02)$.

\section{CONCLUSIONS}

Mycophenolate mofetil was superior to azathioprine in maintaining a renal response to treatment and in preventing relapse in patients with lupus nephritis who had a response to induction therapy. (Funded by Vifor Pharma [formerly Aspreva]; ALMS ClinicalTrials.gov number, NCT00377637.) 
YSTEMIC LUPUS ERYTHEMATOSUS IS AN autoimmune disorder often characterized by the development of glomerulonephritis. ${ }^{1} \mathrm{Re}-$ nal involvement remains the strongest predictor of morbidity and mortality among patients with lupus, ${ }^{1}$ and despite improvements in the management of lupus, the incidence of end-stage renal disease has not declined. ${ }^{2}$ Management of lupus nephritis consists of induction therapy to achieve remission and long-term maintenance therapy to prevent relapse, progression to end-stage renal disease, and death. ${ }^{3}$ However, the options for longterm therapy remain controversial. Treatment options include glucocorticoids and the immunosuppressive agents cyclophosphamide, azathioprine, and mycophenolate mofetil. These drugs have considerable toxicity and are not effective in all patients. ${ }^{4,5}$

There has been mounting interest in the use of mycophenolate mofetil as induction therapy, maintenance therapy, or both for patients with lupus nephritis. ${ }^{6-12}$ The Aspreva Lupus Management Study (ALMS), which examined the efficacy and safety of induction therapy with mycophenolate mofetil as compared with intravenous cyclophosphamide (plus standardized tapering of glucocorticoid therapy) in patients with active lupus nephritis (renal-biopsy active class III, IV, or $\mathrm{V}^{13-16}$ [according to the International Society of $\mathrm{Ne}$ phrology and Renal Pathology Society 2003 Classification of Lupus Nephritis ${ }^{17}$ ), showed no significant difference between the two drugs. ${ }^{13}$ (For a description of the classes of renal biopsy used in this study, see the protocol, available with the full text of this article at NEJM.org.) Here we report the results of a 36-month study of maintenance therapy with either mycophenolate mofetil or azathioprine in patients who had a specified response to treatment during the ALMS induction study.

\section{METHODS}

\section{STUDY DESIGN AND OVERSIGHT}

We conducted a prospective, randomized, doubleblind, double-dummy, phase 3 study comparing mycophenolate mofetil (CellCept, Roche) with azathioprine (Imuran, GlaxoSmithKline) for the maintenance of remission in patients with lupus nephritis. Results of the induction study and detailed descriptions of methods for both phases have been reported previously. ${ }^{13,18}$ The study was conducted in accordance with the Declaration of Helsinki and
Good Clinical Practice principles. The institutional review boards at the participating centers approved the protocol, and all patients or their representatives provided written informed consent. The study was conducted in accordance with the protocol and all amendments.

The study was funded by Vifor Pharma (formerly Aspreva Pharmaceuticals) as part of the Roche-Aspreva rare diseases collaboration. All the investigators have confidentiality agreements with Vifor Pharma. Seven of the academic authors and two authors who are employees of the sponsor conceived of and designed the study, seven of the academic authors collected the data, and all the authors analyzed and interpreted the data. The manuscript was drafted initially by medical writers from Caudex Medical (Oxford, United Kingdom), with funding from Vifor Pharma and in line with guidance from all the authors. It was then amended substantially, critically reviewed, and edited by all the authors, who approved the final version, made the decision to submit the manuscript for publication, and vouch for the completeness and accuracy of the reported data.

\section{STUDY PARTICIPANTS}

Patients 12 to 75 years of age with active class III, IV, or V lupus nephritis who had had a clinical response to either oral mycophenolate mofetil or intravenous cyclophosphamide during the induction study ${ }^{13}$ were randomly assigned (in a 1:1 ratio) to one of these two agents in the maintenance study. The definition of a response was based on investigator judgment but was not necessarily identical to that finally adjudicated by the clinical end-points committee. The maintenance study treatment was randomly assigned by means of a computerized, interactive voice-response system, with stratification according to induction study treatment, race, and classification of renal biopsy.

Patients were followed for 36 months, regardless of events. The vital status of patients who withdrew (with the exception of those who withdrew consent) was obtained annually for 3 years. Patients who completed the 36-month study were contacted 30 days after the last visit to obtain information about any adverse events and use of concomitant medications.

\section{INTERVENTIONS AND SCHEDULE OF ASSESSMENTS}

At the end of the 24-week induction study, patients received oral mycophenolate mofetil (1 g, twice 
daily) or oral azathioprine (2 $\mathrm{mg}$ per kilogram of body weight per day). Patients who were unable to tolerate the target dose or whose weight was below $50 \mathrm{~kg}$ remained in the study if they tolerated a minimum dose of either $1 \mathrm{~g}$ of mycophenolate mofetil per day or $50 \mathrm{mg}$ of azathioprine per day. Temporary reductions below the minimum dose or complete cessation of the drug for no more than 14 days was allowed. Any dose changes were made to both active drug and placebo. Patients could receive prednisone or its equivalent (maximum dose, $10 \mathrm{mg}$ per day), with the dose reduced on the basis of the investigator's judgment.

Patients were assessed at months 0,1 , and 2 and every 3 months thereafter until month 36; at early withdrawal; or at termination due to treatment failure. Selected safety and efficacy evaluations were made at every visit, and patients were contacted by telephone monthly to obtain information about use of concomitant medications. Changes in the doses of angiotensin-converting-enzyme inhibitors, angiotensin-receptor blockers, and nonsteroidal antiinflammatory agents had to be approved by the medical study monitor.

The protocol was amended in April 2009, after discussion with representatives of the European and U.S. health authorities, to make the primary end point more specific for events related to lupus nephritis and to provide a broader definition of treatment failure (the original primary end point was included as a key secondary end point). (The protocol and all amendments can be found at NEJM.org.)

\section{OUTCOMES}

The primary efficacy end point was the time to treatment failure, measured as the time until the first event and defined as death, end-stage renal disease, sustained doubling of the serum creatinine level, renal flare (proteinuric or nephritic), or the need for rescue therapy (glucocorticoids, plasmapheresis, intravenous immune globulin, or immunosuppressive drugs not specified in the protocol) in the event of exacerbation or deterioration of lupus nephritis. A more detailed description of the study outcomes has been reported previously ${ }^{18}$ and is provided in the Supplementary Appendix, available at NEJM.org. Proteinuric renal flare was defined as a doubling of the urinary protein:creatinine ratio and proteinuria ( $\geq 1 \mathrm{~g}$ of protein per 24 hours in patients with urinary protein clearance of $\leq 0.5 \mathrm{~g}$ per 24 hours at the end of induction, and $\geq 2 \mathrm{~g}$ per 24 hours in patients with urinary protein clearance of $>0.5 \mathrm{~g}$ per 24 hours at the end of induction). Nephritic renal flare was defined as an increase of $25 \%$ or more in the lowest serum creatinine level during the period from screening to the end of induction, plus one or more of the following findings: simultaneous doubling of urinary protein clearance, reaching a minimum of $2 \mathrm{~g}$ per 24 hours (or the urinary protein:creatinine ratio equivalent); new or increased hematuria ( $\geq 5$ red cells per highpower field or $\geq 2+$ on a dipstick test for blood); or the appearance of cellular casts.

Key secondary end points included the time to the event for each component of treatment failure, the time to treatment failure defined more broadly (i.e., the components of the primary end point, major extrarenal flare, or withdrawal for any reason), suspected renal flare, complete renal remission (urinary protein clearance, $<500 \mathrm{mg}$ per 24 hours; absence of hematuria and cellular casts; and improved or stable serum creatinine $\pm 25 \%$ of baseline), combined renal and extrarenal remission (absence of a British Isles Lupus Assessment Group category A score [severe lupus flare] in one extrarenal system or lack of a concurrent category B score [moderate lupus flare] in three extrarenal systems ${ }^{19}$ ), and immunologic variables (C3, C4, and anti-double-stranded DNA antibodies).

Safety assessments included clinical measurements (hematologic, serum chemical, and hormonal tests and urinalysis), adverse events, physical examination, vital signs, electrocardiographic studies, and assessment of prior and concomitant medications. As specified by the protocol, to avoid bias, the independent clinical end-points committee, whose members were unaware of the treatment assignments and adverse-event listings, reviewed all results and adjudicated each component of the primary end point and several secondary end points (i.e., suspected renal flare, major or suspected extrarenal flare, and complete extrarenal remission).

\section{STATISTICAL ANALYSIS}

We calculated that we would need to assign 114 patients to each group for the maintenance study to have $80 \%$ or higher power to detect differences between treatments, assuming a failure rate at 3 years of $37.5 \%$ with azathioprine and $20.0 \%$ with mycophenolate mofetil (hazard ratio with mycophenolate mofetil, 0.475), with the use of a logrank test at a two-sided significance level of 0.05.

The primary efficacy analysis was an intentionto-treat analysis that included all patients who 


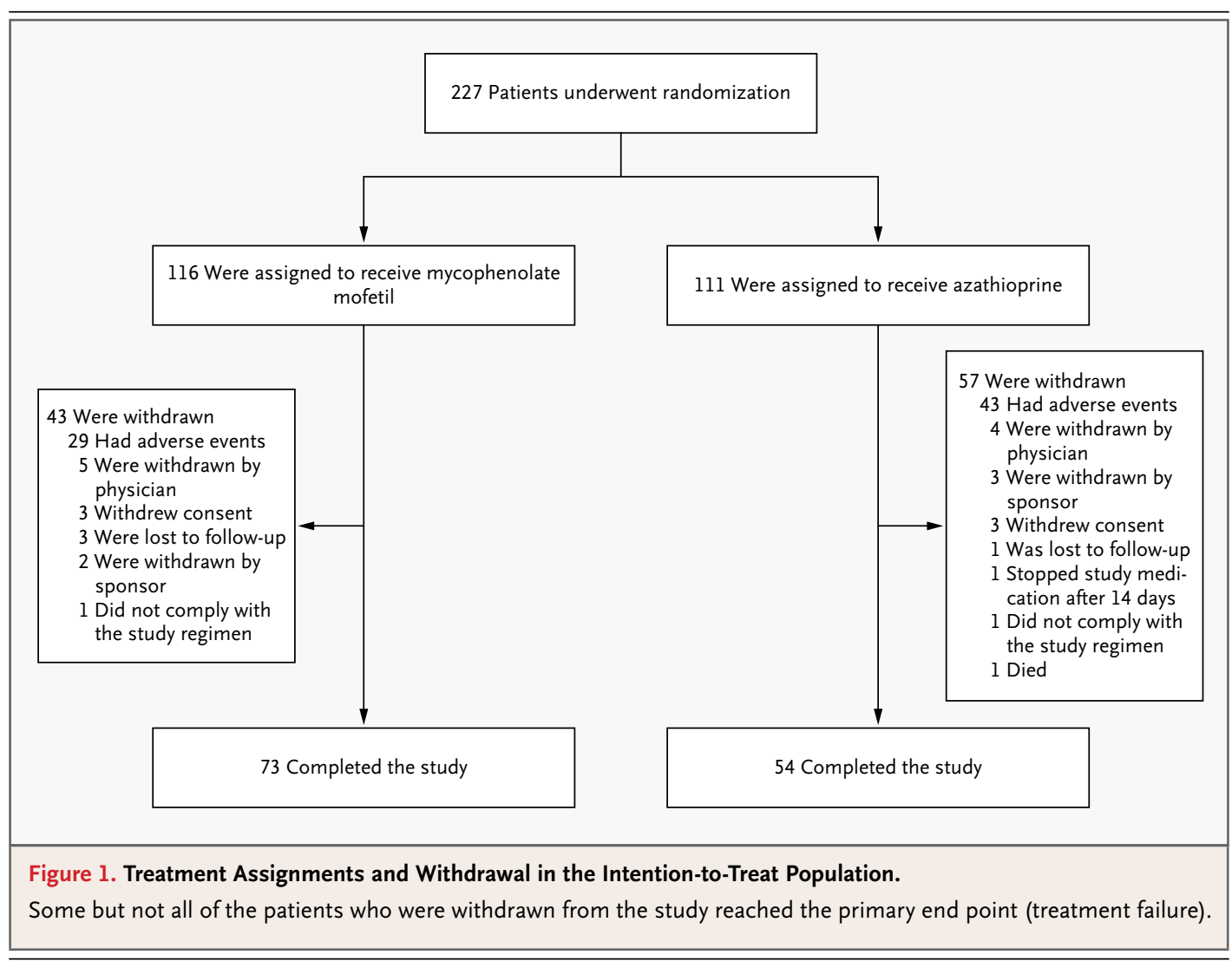

were randomly assigned to the maintenance study and underwent at least one efficacy assessment. The safety population comprised all patients who received at least one dose of study medication and underwent at least one safety assessment. Treatment groups were compared with the use of Kaplan-Meier survival estimates for the time to treatment failure for each patient, with censoring of data for patients who withdrew before the end of the study. ${ }^{20}$ Between-group differences in survival curves were assessed with the use of a logrank test. ${ }^{21}$

The magnitude of the treatment effect was estimated by means of the hazard ratio obtained from an unadjusted Cox model. Hazard ratios were also estimated in subgroups stratified according to induction therapy, race, and geographic region. The overall incidence of events and the event rates per 100 person-years for both treatments are presented within subgroups. Secondary efficacy variables were analyzed by calculating hazard ratios from unadjusted Cox models. Sensitivity analyses for the primary end point were conducted, with adjustment for covariates. Testing at the significance level of 0.05 was applied to the primary efficacy analysis and to any key secondary efficacy analyses (with no adjustments for multiple comparisons). Safety variables were analyzed descriptively, with a between-group comparison of proportions of patients with adverse events.

Statistical analyses were performed with the use of SAS software, version 9.2 (SAS Institute). For analyses of changes from baseline over time (at the time of the last visit), the last-observation-carriedforward method was used.

\section{RESULTS}

\section{PATIENTS}

A total of 227 patients were enrolled between January 25, 2006, and March 19, 2007 (72 in Asia, 60 in Latin America, 47 in North America, 40 in Europe, 5 in South Africa, and 3 in Australia). Of the patients in this intention-to-treat population, 116 were randomly assigned to mycophenolate mofetil and 111 to azathioprine. A total of $127 \mathrm{pa}-$ tients $(55.9 \%)$ completed the 36 months of treatment: $73(62.9 \%)$ in the mycophenolate mofetil group and 54 (48.6\%) in the azathioprine group (Fig. 1). The main reasons for early withdrawal 
in both groups were adverse events and flares of lupus nephritis. There were no significant differences between the groups with respect to demographic or disease characteristics (Table 1).

\section{IMMUNOSUPPRESSIVE THERAPIES}

The safety population comprised 226 patients, of whom 115 received mycophenolate mofetil and 111 received azathioprine. Mean $( \pm S D)$ doses of mycophenolate mofetil and azathioprine were $1.87 \pm 0.43 \mathrm{~g}$ and $119.7 \pm 47.91 \mathrm{mg}$, respectively. The proportion of patients whose average daily dose was $80 \%$ or more of the target dose was $79.7 \%$ for mycophenolate mofetil and $82.6 \%$ for azathioprine. In a subgroup analysis of patients for whom treatment failed, the mean doses were $1.89 \pm 0.52 \mathrm{~g}$ and $123.3 \pm 49.73 \mathrm{mg}$, respectively. The mean treatment duration was $834.4 \pm 363$ days for mycophenolate mofetil and $727.2 \pm 405$ days for azathioprine. The majority of patients received $10 \mathrm{mg}$ of prednisone per day or less throughout the study: 104 patients (90.4\%) and 96 patients (86.5\%) in the mycophenolate mofetil and azathioprine groups, respectively.

\section{STUDY END POINTS}

Mycophenolate mofetil was significantly superior to azathioprine with respect to the primary end point, time to treatment failure (hazard ratio for treatment failure, 0.44; 95\% confidence interval [CI], 0.25 to $0.77 ; \mathrm{P}=0.003$ ) (Fig. 2). Overall observed rates of treatment failure were $16.4 \%$ (19 of 116 patients) in the mycophenolate mofetil group and $32.4 \%$ (36 of 111) in the azathioprine group. The superiority of mycophenolate mofetil was consistent, regardless of induction therapy, race, and geographic region (Fig. 3).

Mycophenolate mofetil was also superior to azathioprine with respect to individual compo-

\begin{tabular}{|c|c|c|}
\hline Characteristic & $\begin{array}{l}\text { Mycophenolate Mofetil } \\
(\mathrm{N}=116)\end{array}$ & $\begin{array}{l}\text { Azathioprine } \\
(\mathrm{N}=111)\end{array}$ \\
\hline \multicolumn{3}{|l|}{ Sex - no. of patients (\%) } \\
\hline Male & $17(14.7)$ & $15(13.5)$ \\
\hline Female & $99(85.3)$ & $96(86.5)$ \\
\hline \multicolumn{3}{|l|}{ Race - no. of patients (\%) † } \\
\hline White & $48(41.4)$ & $51(45.9)$ \\
\hline Black & $12(10.3)$ & $11(9.9)$ \\
\hline Asian & $39(33.6)$ & $37(33.3)$ \\
\hline Other & $17(14.7)$ & $12(10.8)$ \\
\hline \multicolumn{3}{|l|}{ Ethnic group - no. of patients (\%) } \\
\hline Hispanic & $36(31.0)$ & $41(36.9)$ \\
\hline Non-Hispanic & $80(69.0)$ & $70(63.1)$ \\
\hline Age at time of informed consent $-\mathrm{yr}$ & $31.8 \pm 10.59$ & $31.0 \pm 10.77$ \\
\hline Weight - kg & $64.7 \pm 16.64$ & $64.0 \pm 16.12$ \\
\hline Duration of lupus nephritis $-\mathrm{yr}$ & $3.1 \pm 4.64$ & $2.9 \pm 4.20$ \\
\hline Duration of systemic lupus erythematosus - yrt; & $5.8 \pm 6.41$ & $4.9 \pm 5.62$ \\
\hline \multicolumn{3}{|l|}{ Renal-biopsy active class - no. of patients (\%) $\mathbb{S}$} \\
\hline $\mathrm{III}$ or III $+\mathrm{V}$ & $17(14.7)$ & $12(10.8)$ \\
\hline IV or IV + V & $81(69.8)$ & $82(73.9)$ \\
\hline V only & $18(15.5)$ & $17(15.3)$ \\
\hline Urinary protein $-\mathrm{mg} / 24 \mathrm{hr}$ & $906 \pm 819.93$ & $820.0 \pm 754.33$ \\
\hline Urinary protein:creatinine ratio & $0.97 \pm 1.413$ & $0.75 \pm 0.830$ \\
\hline Serum creatinine $-\mathrm{mg} / \mathrm{dl} \|$ & $0.82 \pm 0.24$ & $0.90 \pm 0.38$ \\
\hline $\mathrm{eGFR}-\mathrm{ml} / \mathrm{min} / 1.73 \mathrm{~m}^{2 * * *}$ & $104.9 \pm 43.13$ & $98.6 \pm 38.22$ \\
\hline
\end{tabular}




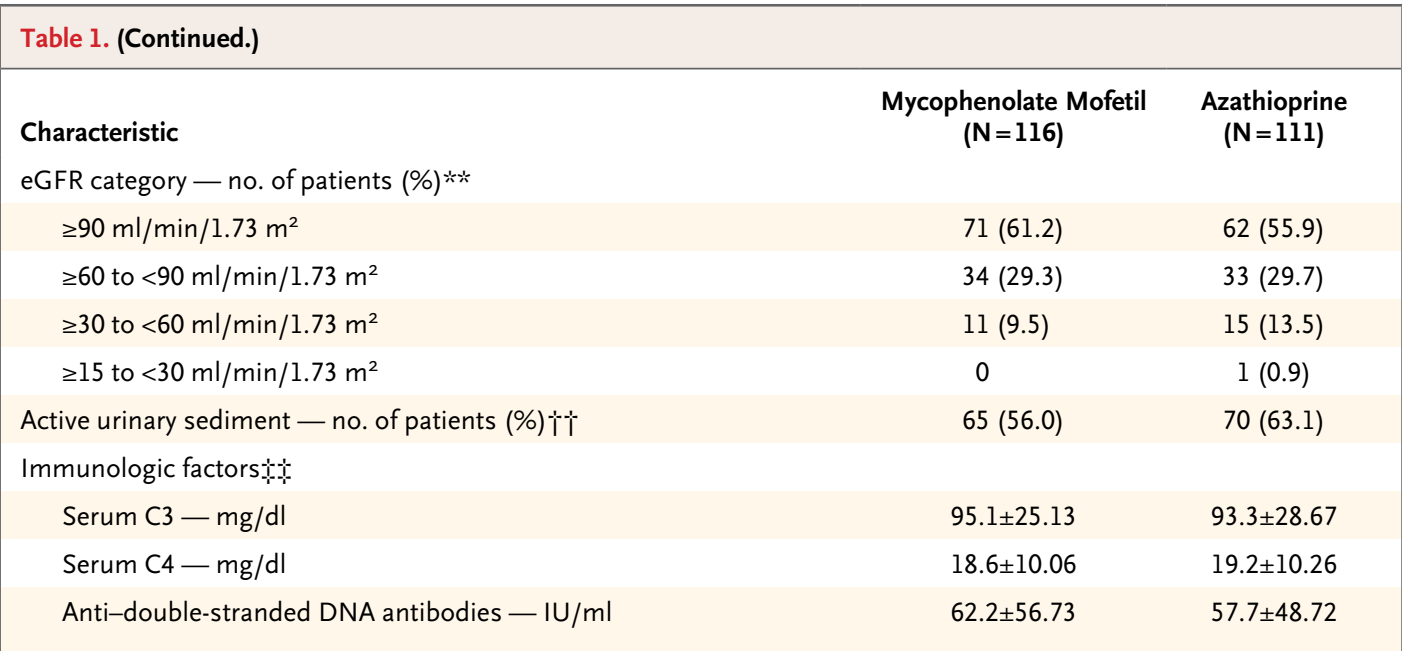

* Plus-minus values are means \pm SD. There were no significant differences between the two treatment groups with respect to any of the demographic or baseline clinical characteristics. Demographic characteristics (sex, race, ethnic group, and age at time of consent) and renal-biopsy class were determined only at the start of induction therapy. Durations refer to the time since the start of maintenance therapy. Reference ranges are as follows: urinary protein:creatinine ratio (mg:mg), 0.015 to 0.220 ; serum creatinine, 0.49 to $1.40 \mathrm{mg}$ per deciliter; estimated glomerular filtration rate (eGFR), 90 to $120 \mathrm{ml}$ per minute per $1.73 \mathrm{~m}^{2}$ of body-surface area; and anti-double-stranded DNA antibodies, <30 IU per milliliter.

$\dagger$ Race was self-reported.

+ Duration refers to the length of time the disease had been present at the start of induction therapy.

$\int$ The renal-biopsy specimens were classified according to the International Society of Nephrology and Renal Pathology Society 2003 Classification of Lupus Nephritis ${ }^{17}$ and are described in the protocol at NEJM.org.

9 The unit of measurement was mg:mg.

|| To convert the values for creatinine to micromoles per liter, multiply by 88.4 .

*** The estimated glomerular filtration rate is calculated with the use of the Modification of Diet in Renal Disease formula ${ }^{17,22}$. $186 \times(\text { serum creatinine in } \mathrm{mg} / \mathrm{dl})^{-1.154} \times$ age $^{-0.203} \times 1.21$ if black $\times 0.742$ if female.

t个 An active urinary sediment is defined as the presence of more than 5 white cells per high-power field, more than 5 red cells per high-power field, a dipstick result of 2+ or more for blood, and red-cell casts in the absence of infection or other causes. tr Values are geometric means.

nents of treatment failure, including the time to renal flare (hazard ratio for flare, $0.50 ; 95 \% \mathrm{CI}$, 0.26 to $0.93 ; \mathrm{P}=0.03)$. Renal flare occurred in 15 of 116 patients (12.9\%) given mycophenolate mofetil versus 26 of 111 patients (23.4\%) given azathioprine (Fig. 2). The time to rescue therapy for lupus nephritis was also longer with mycophenolate mofetil than with azathioprine (hazard ratio, $0.39 ; 95 \% \mathrm{CI}, 0.18$ to $0.87 ; \mathrm{P}=0.02$ ); the rates of rescue were $7.8 \%$ ( 9 of 116 patients) and $17.1 \%$ (19 of 111), respectively.

Mycophenolate mofetil was significantly superior to azathioprine for the key secondary end points of the broader definition of treatment failure (rate, $42.2 \%$ [ 49 of 116 patients] in the mycophenolate mofetil group vs. $56.8 \%$ [63 of 111] in the azathioprine group; hazard ratio, $0.66 ; 95 \% \mathrm{CI}$, 0.46 to $0.97 ; \mathrm{P}=0.03$ ), and the time to documented or suspected renal flare (rate, $21.6 \%$ [25 of 116 patients] vs. $36.0 \%$ [ 40 of 111], respectively; hazard ratio, 0.56 ; $95 \% \mathrm{CI}, 0.35$ to $0.89 ; \mathrm{P}=0.01)$. All other components of the primary efficacy end point showed a numerical benefit in favor of mycophenolate mofetil, including the time to end-stage renal disease (rate, $0 \%$ [0 of 116 patients] in the mycophenolate mofetil group vs. $2.7 \%$ [3 of 111] in the azathioprine group; $\mathrm{P}=0.07$ ), and the time to sustained doubling of the serum creatinine level (rate, $0.9 \%$ [1 of 116 patients] vs. $4.5 \%$ [5 of 111], respectively; $\mathrm{P}=0.07)$. The benefit of mycophenolate mofetil was maintained after adjustments were made for the serum creatinine level $(\mathrm{P}=0.004)$ and the estimated glomerular filtration rate at the end of induction therapy $(\mathrm{P}=0.004)$. The rates of major extrarenal flares were low: 6.9\% (8 of 116 patients) in the mycophenolate mofetil group and $6.3 \%$ (7 of 111) in the azathioprine group. The time to a major extrarenal flare did not differ significantly between the two groups $(\mathrm{P}=0.94)$.

During the first 3 months, the geometric mean 


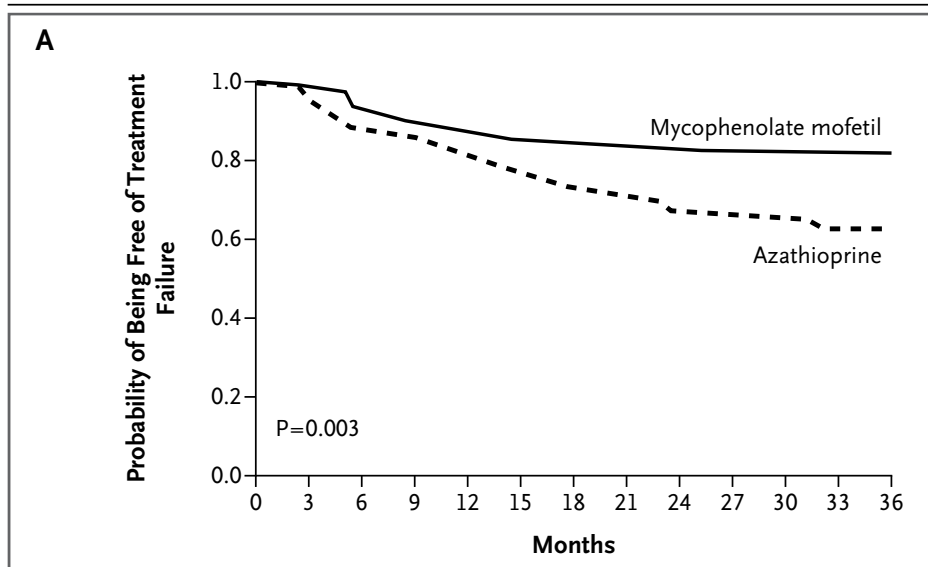

No. at Risk

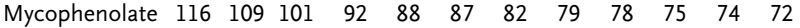
mofetil

$\begin{array}{lllllllllllll}\text { Azathioprine } & 111 & 101 & 88 & 81 & 77 & 70 & 64 & 61 & 58 & 56 & 52 & 51\end{array}$

B

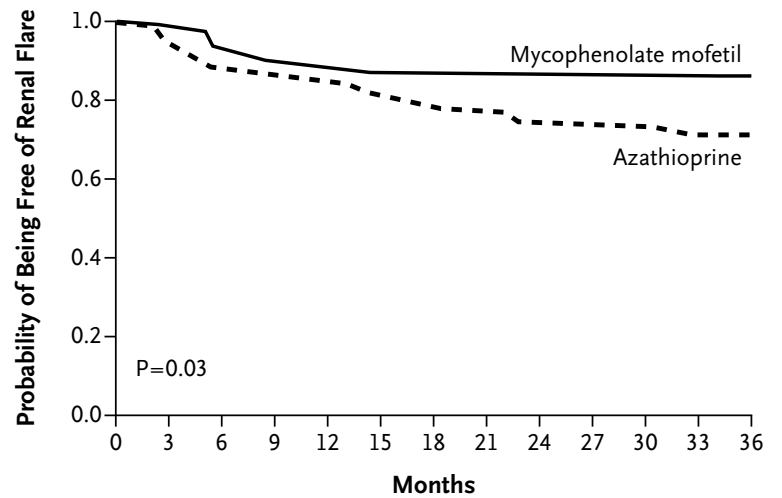

No. at Risk

$\begin{array}{lllllllllllll}\text { Mycophenolate } & 116 & 109 & 102 & 92 & 89 & 88 & 82 & 80 & 78 & 75 & 74 & 73\end{array}$ mofetil

$\begin{array}{lllllllllllll}\text { Azathioprine } & 111 & 101 & 89 & 82 & 77 & 71 & 65 & 62 & 60 & 58 & 56 & 54\end{array}$

Figure 2. Kaplan-Meier Curves for Time to Treatment Failure and Time to Renal Flare.

The intention-to-treat population comprised 227 patients, of whom 116 were given mycophenolate mofetil and 111 were given azathioprine. Panel $A$ shows the time to treatment failure, and Panel $\mathrm{B}$ shows the time to renal flare.

titers of $\mathrm{C} 3$ and $\mathrm{C} 4$ in serum were lower in the azathioprine group than those in the mycophenolate mofetil group, and they remained lower throughout the study. Over time, there was a trend toward greater decreases in geometric mean titers of anti-double-stranded DNA antibodies in the patients treated with mycophenolate mofetil.

\section{SAFETY AND TOLERABILITY}

The incidence of adverse events during treatment was similar in the two groups: 98.3\% (113 of 115 patients given mycophenolate mofetil) and $97.3 \%$ (108 of 111 patients given azathioprine, $\mathrm{P}=0.68$ ) (Table 2). For additional details, see the Supplementary Appendix.

Infections were the most common adverse events in both groups, with a rate of: 79.1\% (91 of 115 patients) in the mycophenolate mofetil group and $78.4 \%$ (87 of 111) in the azathioprine group. The rate of serious infections was low in both groups: $9.6 \%$ (11 of 115 patients) in the mycophenolate mofetil group and $11.7 \%$ (13 of 111) in the azathioprine group.

The proportion of patients with adverse events leading to withdrawal was higher with azathioprine (39.6\% [ 44 of 111 patients]) than with mycophenolate mofetil (25.2\% [29 of 115], $\mathrm{P}=0.02)$. Fewer patients in the mycophenolate mofetil group than in the azathioprine group had at least one serious adverse event, although the difference was not significant $(23.5 \%$ [27 of 115 patients] vs. $33.3 \%$ [37 of 111], $\mathrm{P}=0.11$ ). During the study, one death occurred in the azathioprine group and was due to a traffic accident. One case of cancer (uterine carcinoma in situ) was diagnosed in the azathioprine group.

\section{DISCUSSION}

There are few data concerning maintenance therapy for lupus nephritis. ${ }^{8,11}$ A suboptimal response to treatment in patients with class III, IV, or V lupus nephritis increases the risk of end-stage renal disease. ${ }^{23}$ The early National Institutes of Health (NIH) regimen of intravenous cyclophosphamide became the standard of care in many countries but was challenged by a long-term maintenance trial showing that oral mycophenolate mofetil and oral azathioprine were each superior to the standard 2-year NIH cyclophosphamide regimen. ${ }^{11}$ In an open-label study of maintenance therapy in 105 patients with lupus nephritis, Houssiau et al. ${ }^{8}$ reported no significant difference in relapse rates between patients who were randomly assigned to receive azathioprine and those assigned to receive mycophenolate mofetil. Renal flares were observed in 10 of 53 patients given mycophenolate mofetil (18.9\%), as compared with 13 of 52 patients given azathioprine (25.0\%).

In our study, mycophenolate mofetil was superior to azathioprine in maintaining the renal response and in preventing relapse in patients with active lupus nephritis who had had a response 


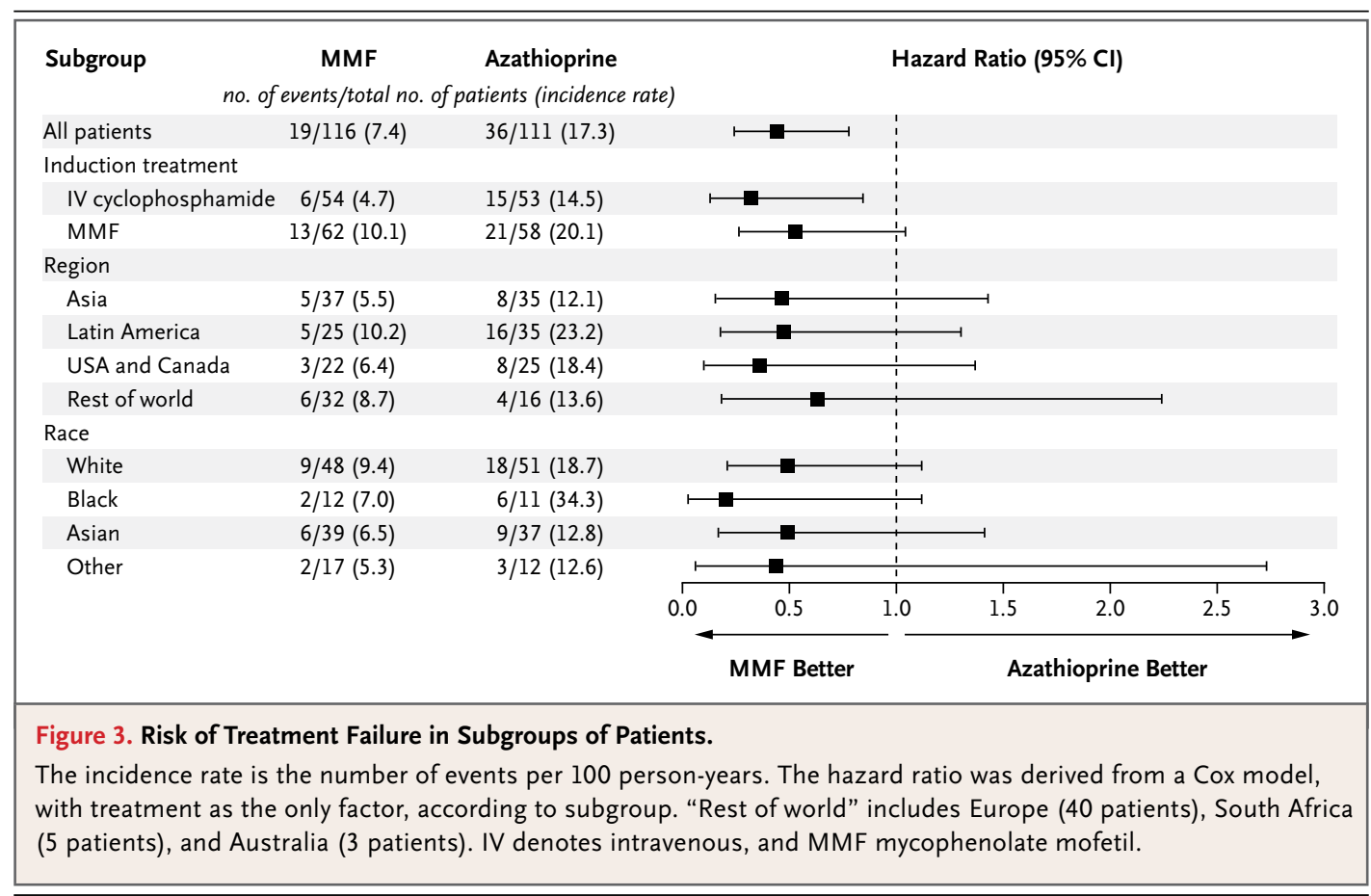

to induction therapy with either mycophenolate mofetil or cyclophosphamide. There were few nephritic flares, an expected finding, since the majority of patients entered the induction study with ongoing proteinuria rather than clinical nephritis. Even when broader end-point criteria were applied, mycophenolate mofetil proved superior to azathioprine. The results for other key secondary end points also confirmed the superiority of mycophenolate mofetil over azathioprine - findings that were consistent regardless of the induction therapy received, race, and geographic region.

Rates of complete remission at the end of induction therapy were low $(8.6 \%$ with mycophenolate mofetil and $8.1 \%$ with cyclophosphamide). ${ }^{13}$ Although the aim of the study was to prevent relapse, patients in both treatment groups continued to have improvement, with complete remission eventually achieved in $62.1 \%$ of patients in the mycophenolate mofetil group (72 of 116) and in $59.5 \%$ of those in the azathioprine group (66 of 111). This finding suggests that the distinction between induction therapy and maintenance therapy in patients with lupus nephritis may be an artificial one.

Little extrarenal lupus activity was observed in either group at the start of maintenance therapy or at the end of the study, which probably reflects the requirement of a clinical response for entry into the maintenance study. Adverse events were common in both groups and were consistent with previous findings. ${ }^{8,11,13}$ One death occurred in the azathioprine group and the frequency of serious infections was low in both groups. Azathioprine was associated with adverse hematologic events (a finding that is consistent with its known safety profile), and serious adverse events and withdrawals from the study were also more common in this treatment group.

This study was not powered to draw conclusions about subsets of patients. Few repeat renal biopsies were performed during the study, which also limits the ability to draw conclusions about possible treatment benefits with respect to active disease or chronic renal damage.

Although our trial included more patients and was substantially longer than many of the controlled trials involving patients with lupus nephritis, ${ }^{6-8,11,24}$ potential outcomes that might appear more frequently after 5 to 20 years (e.g., cardiovascular complications and end-stage renal disease) cannot be determined, since no further follow-up study is planned. The length of time that treatment with mycophenolate mofetil needs to be continued is unknown; hence, improved biomarkers of response are needed to distinguish disease remission from remission that occurs while the patient is receiving treatment. 


\begin{tabular}{|c|c|c|c|}
\hline \multirow[t]{2}{*}{ Event } & $\begin{array}{l}\text { Mycophenolate Mofetil } \\
(\mathrm{N}=115) \dagger\end{array}$ & $\begin{array}{l}\text { Azathioprine } \\
(N=111)\end{array}$ & $P$ Value \\
\hline & \multicolumn{2}{|c|}{ no. of patients (\%) } & \\
\hline All adverse eventst & $113(98.3)$ & $108(97.3)$ & 0.68 \\
\hline All serious adverse events & $27(23.5)$ & $37(33.3)$ & 0.11 \\
\hline All adverse events leading to withdrawal & $29(25.2)$ & $44(39.6)$ & 0.02 \\
\hline \multicolumn{4}{|l|}{ Serious adverse events not related to lupus } \\
\hline Death & 0 & $1(0.9)$ & 0.49 \\
\hline Malignant conditions & 0 & $1(0.9)$ & 0.49 \\
\hline Pneumonia & $2(1.7)$ & $1(0.9)$ & 1.00 \\
\hline Appendicitis & 0 & $2(1.8)$ & 0.24 \\
\hline Leukopenia & 0 & $4(3.6)$ & 0.06 \\
\hline Systemic lupus erythematosus & $3(2.6)$ & $2(1.8)$ & 1.00 \\
\hline Lupus nephritis & $1(0.9)$ & $5(4.5)$ & 0.11 \\
\hline
\end{tabular}

* The terms used to describe the events are those preferred by the Medical Dictionary for Regulatory Activities, version 12.0. Only adverse events that occurred during treatment are listed; these include any nonserious adverse event that occurred between the date of the first dose and the date of the last visit plus 14 days and any serious adverse event that occurred after the date of the first dose. Multiple occurrences of the same adverse event in one person were counted only once. $\dagger$ One patient in this group was randomly assigned to treatment in error and did not receive the study drug.

$\checkmark$ This category includes all patients who had at least one adverse event.

Patients who had a clinical response after 6 months of induction therapy were included in this maintenance study. Those patients whose disease was more difficult to treat, therefore, may have been excluded. Since many black patients did not meet the clinical response criteria, few were included in the maintenance trial. Most patients reached their target dose and remained in the maintenance study for the full 36 months. The study was adequately powered to assess the primary composite outcome, which was achieved, with results consistent across the treatment groups.

We conclude that mycophenolate mofetil is superior to azathioprine in maintaining the renal response to treatment and in preventing relapse in patients with active lupus nephritis who have had a clinical response to induction therapy with either mycophenolate mofetil or intravenous cyclophosphamide.

Supported by Vifor Pharma (formerly Aspreva Pharmaceuticals) as part of the Roche-Aspreva rare diseases collaboration.

Disclosure forms provided by the authors are available with the full text of this article at NEJM.org.

We thank Steven Nettler, M.P.H. (Vifor Pharma), for statistical support and interpretation of the data; Nicola West, B.Sc. (Caudex Medical), for assisting in the preparation of an earlier draft of the manuscript, drafting earlier versions of the tables and figures, and collating author comments; and Christine Groves, B.Sc. (Caudex Medical), for assisting the authors in addressing comments from reviewers.
REFERENCES

1. Mills JA. Systemic lupus erythematosus. N Engl J Med 1994;330:1871-9.

2. Ward MM. Changes in the incidence of end-stage renal disease due to lupus nephritis, 1982-1995. Arch Intern Med 2000;160:3136-40.

3. Ponticelli C, Moroni G. Flares in lupus nephritis: incidence, impact on renal survival and management. Lupus 1998;7:635-8. 4. Flanc RS, Roberts MA, Strippoli GF, Chadban SJ, Kerr PG, Atkins RC. Treatment of diffuse proliferative lupus nephritis: a meta-analysis of randomized con- trolled trials. Am J Kidney Dis 2004;43: 197-208.

5. Bomback AS, Appel GB. Updates on the treatment of lupus nephritis. J Am Soc Nephrol 2010;21:2028-35.

6. Chan TM, Li FK, Tang CS, et al. Efficacy of mycophenolate mofetil in patients with diffuse proliferative lupus nephritis. N Engl J Med 2000;343:1156-62.

7. Ginzler EM, Dooley MA, Aranow C, et al. Mycophenolate mofetil or intravenous cyclophosphamide for lupus nephritis. N Engl J Med 2005;353:2219-28.
8. Houssiau FA, D'Cruz D, Sangle S, et al. Azathioprine versus mycophenolate mofetil for long-term immunosuppression in lupus nephritis: results of the MAINTAIN nephritis trial. Ann Rheum Dis 2010;69: 2083-9.

9. Sahin GM, Sahin S, Kiziltas S, Masatlioglu S, Oguz F, Ergin H. Mycophenolate mofetil versus azathioprine in the maintenance therapy of lupus nephritis. Ren Fail 2008;30:865-9.

10. Chan TM. Lupus nephritis: induction therapy. Lupus 2005;14:Suppl 1:S27-S32. 
11. Contreras G, Pardo V, Leclercq B, et al. Sequential therapies for proliferative lupus nephritis. N Engl J Med 2004;350: 971-80.

12. Ong LM, Hooi LS, Lim TO, et al. Randomized controlled trial of pulse intravenous cyclophosphamide versus mycophenolate mofetil in the induction therapy of proliferative lupus nephritis. Nephrology (Carlton) 2005;10:504-10.

13. Appel GB, Contreras G, Dooley MA, et al. Mycophenolate mofetil versus cyclophosphamide for induction treatment of lupus nephritis. J Am Soc Nephrol 2009; 20:1103-12.

14. Ginzler E, Wofsy D, Isenberg D, Gordon C, Lisk L, Dooley MA. Nonrenal disease activity following mycophenolate mofetil or intravenous cyclophosphamide as induction treatment for lupus nephritis: findings in a multicenter, prospective, randomized, open-label, parallel-group clinical trial. Arthritis Rheum 2010;62:211-21. [Erratum, Arthritis Rheum 2010;62:3005.] 15. Isenberg D, Appel GB, Contreras G, et al. Influence of race/ethnicity on response to lupus nephritis treatment: the ALMS study. Rheumatology (Oxford) 2010;49: 128-40.

16. Isenberg D, Gordon C, Merrill J, Urowitz M. New therapies in systemic lupus erythematosus - trials, troubles and tribulations. . . . working towards a solution. Lupus 2008;17:967-70.

17. Weening JJ, D'Agati VD, Schwartz $\mathrm{MM}$, et al. The classification of glomerulonephritis in systemic lupus erythematosus revisited. J Am Soc Nephrol 2004;15: 241-50.

18. Sinclair A, Appel G, Dooley MA, et al. Mycophenolate mofetil as induction and maintenance therapy for lupus nephritis: rationale and protocol for the randomized, controlled Aspreva Lupus Management Study (ALMS). Lupus 2007;16:972-80.

19. Hay EM, Bacon PA, Gordon C, et al. The BILAG index: a reliable and valid instrument for measuring clinical disease activity in systemic lupus erythematosus. Q J Med 1993;86:447-58.
20. Kaplan EL, Meier P. Nonparametric estimation from incomplete observations. J Am Stat Assoc 1958;53:457-81.

21. Mantel N. Evaluation of survival data and two new rank order statistics arising in its consideration. Cancer Chemother Rep 1966;50:163-70.

22. Levey AS, Coresh J, Greene T, et al. Using standardized serum creatinine values in the Modification of Diet in Renal Disease study equation for estimating glomerular filtration rate. Ann Intern Med 2006;145:247-54. [Erratum, Ann Intern Med 2008;149:519.]

23. Ponticelli C, Glassock RJ, Moroni G. Induction and maintenance therapy in proliferative lupus nephritis. J Nephrol 2010;23:9-16

24. Chan TM, Tse KC, Tang CS, Mok MY, Li FK. Long-term study of mycophenolate mofetil as continuous induction and maintenance treatment for diffuse proliferative lupus nephritis. J Am Soc Nephrol 2005; 16:1076-84

Copyright (c) 2011 Massachusetts Medical Society.

CLINICAL TRIAL REGISTRATION

The Journal requires investigators to register their clinical trials in a public trials registry. The members of the International Committee of Medical Journal Editors (ICMJE) will consider most reports of clinical trials for publication only if the trials have been registered.

Current information on requirements and appropriate registries is available at www.icmje.org/faq_clinical.html. 